\title{
VOLTAGE CONTROLLED AMPLIFIER WITH LINEARIZED RESPONSE TO THE CONTROLLING VOLTAGE
}

\author{
Martin Pospisilik ${ }^{\mathrm{a}}$, Rui Miguel Soares Silva ${ }^{\mathrm{b}}$, Milan Adamek ${ }^{\mathrm{a}}$ \\ ${ }^{a}$ Faculty of Applied Informatics, Tomas Bata University, Nad Stranemi 4511, Zlin, Czech Republic \\ ${ }^{b}$ Polytechnic Institute of Beja, Laboratory UbiNET, Rua Pedro Soares, Beja, Portugal
}

\begin{abstract}
In this paper a design of a voltage controlled amplifier is described together with its practical realization and measured parameters. The amplifier is supposed to be employed in audio signal processing systems, for example volume expanders, etc. The design was created with the aid of several software supports as Maple for mathematical description of the circuit or Multisim for complex analysis of the amplifier's behaviour. The verification of the design was done by practical construction and performance measurements. The discussion on the differences between the simulated and really obtained results is also included in the framework of this paper.
\end{abstract}

Keyword: Dynamics expansion; dynamics compression; voltage controlled amplifier; linearization
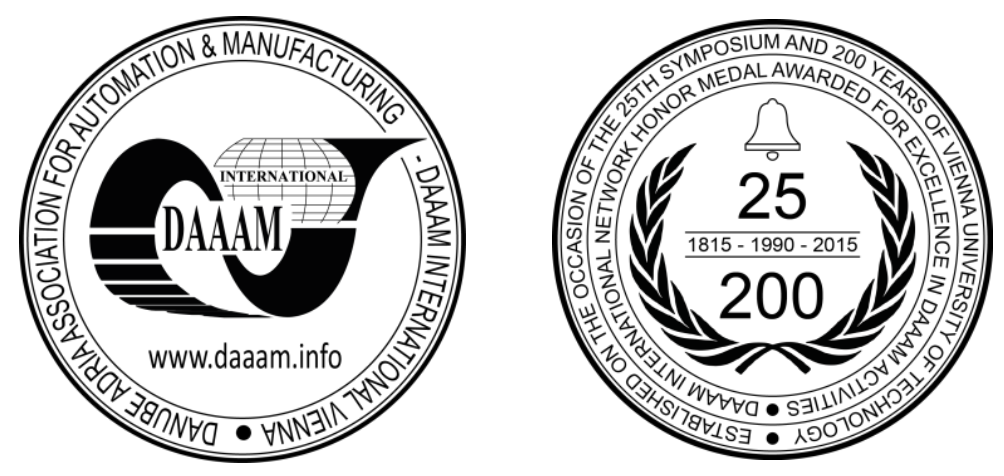

This Publication has to be referred as: Pospisilik, M[artin]; Silva, R[ui] M[iguel Soares] \& Adamek, M[ilan] (2016). Voltage Controlled Amplifier with Linearized Response to the Controlling Voltage, Proceedings of the 26th DAAAM International Symposium, pp.0336-0342, B. Katalinic (Ed.), Published by DAAAM International, ISBN 978-3-90273407-5, ISSN 1726-9679, Vienna, Austria

DOI:10.2507/26th.daaam.proceedings.045 


\section{Introduction}

In audio systems, voltage-controlled amplifiers are widely used for various purposes $[1,2,7,8]$. Some of the typical applications are compressors and expanders of dynamics. The amplifier presented in this paper was designed and constructed for the purposes of audio signal dynamics expander with the expansion rate of 3.3:1 (10 dB). One of the basic requirements was to assure that the amplification factor of the circuit is linearly proportional to the controlling voltage. For example, controlling voltage $1.5 \mathrm{~V}$ refers to the amplification factor of 1.5 , controlling voltage of $3 \mathrm{~V}$ refers to the amplification factor of 3 etc. Voltages below $1 \mathrm{~V}$ and above $3.3 \mathrm{~V}$ should be ignored with the amplification factor set to the corresponding limit.

The primary purpose of this project was to create a set of voltage controlled amplifiers to be employed in hardware dynamics expander operating on the same principle as was described in [3].

As it was required to create and test a low-cost and easy to be built functional sample of this amplifier, the designers decided to build a circuit that employs one quad low noise amplifier and a unipolar transistor connected as a driven resistor. The linearity of the unipolar transistor for large audio signals should be ensured by the local feedback according to [4] and the linearity of amplification factor dependence on the control voltage is ensured by using a complementary reference amplifier.

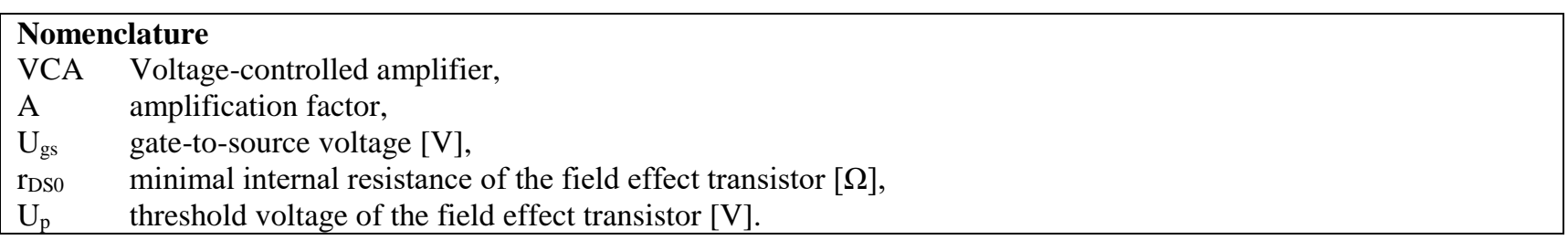

\section{Design description}

The design of the circuit was processed with the aid of several software tools. Transfer functions of the circuit and DC biases were calculated by help of Maple software. The simulation of the whole circuit was processed by Multisim in order to check the functionality of the circuit before the functional sample was created.

\subsection{General description}

The design of the circuit was processed according to the following requirements:

- Input gain variable from - $10 \mathrm{do}+10 \mathrm{~dB}$,

- $\quad$ Nominal input voltage $0 \mathrm{dBu}(0.775 \mathrm{~V})$,

- Overexcitation of the amplifier at least $10 \mathrm{~dB}$,

- Frequency response at least $10 \mathrm{~Hz}$ to $50 \mathrm{kHz}$.

- Input gain controlled by a linear potentiometer.

Considering the maximum amplification factor of $10 \mathrm{~dB}$, minimal voltage swing of $20 \mathrm{~V}$ must be ensured at the output of the circuit. To achieve this, the optimal power supply voltage is around $\pm 15 \mathrm{~V}$. Block diagram of the circuit is depicted in Figure 1.

The gain of the input amplifier is adjustable by means of a linear potentiometer connected in that way so it acted as the logarithmical one. This solution was chosen because linear stereo potentiometers usually embody better matching of channels. Once the level of the signal is adjusted to the desired level, the signal is processed by the voltage controlled amplifier (VCA I in Figure 1). The amplification factor of the VCA I amplifier is set by the DC voltage at the back end of the DC control voltage amplifier. The linear dependence of the amplification factor on the input control voltage is achieved by utilizing the negative feedback from the voltage controlled amplifier VCA II back to the DC control voltage amplifier. Both voltage controlled amplifiers, VCA I and VCA II, are identical.

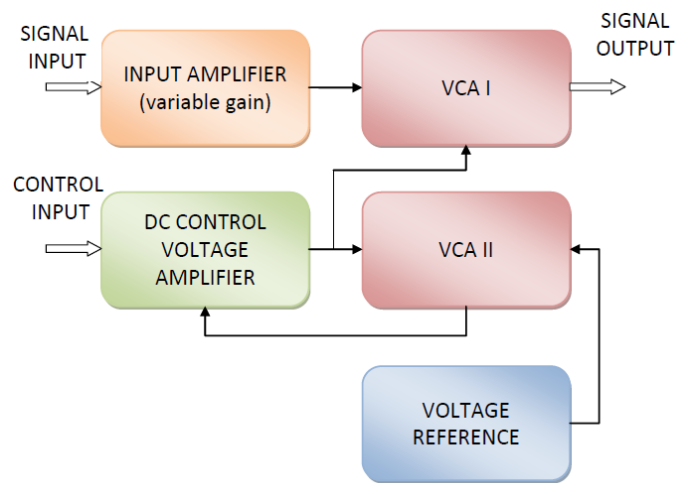

Fig. 1. Block diagram of the voltage controlled amplifier 


\subsection{Detailed description}

The circuit diagram of the functional sample that was finally built for the purposes of practical testing is depicted in Figure 2. In Table 1 the description of the input/output connectors is provided. The need of symmetrical power supply is served by a charge pump with IC1 that produces negative voltage approximately - $15 \mathrm{~V}$. This solution has proven to be successful as the power consumption of the circuit is low. The most important advantage of this solution is a need of non-symmetrical power supply source. The input amplifier (see Figure 1) is based on the operating amplifier IC2C. Its gain is controlled by the linear potentiometer R18. The connection of this preamplifier enables to use the linear potentiometer as the logarithmic one. Assuming the internal gain of the operating amplifier is infinite and its phase shift is negligible, by mathematical analysis of the transfer function of this circuit in Maple it was proven that the gain of the preamplifier can be expressed as follows:

$$
A_{I C 2 C}=\frac{R 16}{R 12+R 16} \cdot\left(1+\frac{x \cdot R 18+R 20}{(1-x) \cdot R 18+R 11}\right)-\frac{x \cdot R 18+R 20}{(1-x) \cdot R 18+R 11}
$$

Where: R11, R12, R16 and R20 are ohmic values of relevant resistors (see Figure 2), R18 is ohmic value of the potentiometer and $\mathrm{x}$ is the potentiometer's slider position, whereby value close to 0 refers to the left limit position of the slider and value close to 1 refers to the right limit position of the slider. The phase of the signal is inverted.

Optimal values of the resistors were found by several mathematical calculations applied to (1) in Maple. For the values indicated in Figure 2, the dependency of amplification factor on setting the potentiometer with a resistance of $100 \mathrm{k} \Omega$ can be seen in Figure 3. The voltage-controlled amplifiers VCAI and VCAII use similar topology, but instead of potentiometers, field effect transistors T1 and T2 are utilized to control the gain. The "signal" amplifier VCAI is based on operating amplifier IC2D while the "reference" amplifier VCAII is based on operating amplifier IC2A [5]. Both of them are identical. The description of VCAI is valid also for the description of VCAII, only device numbers are different. Therefore only a description on VCAI is provided in the text below.

The operating amplifier IC2D is connected as a differential amplifier. Under the assumptions that $\mathrm{Ra}=\mathrm{R} 33=\mathrm{R} 34$ and $\mathrm{Rb}=\mathrm{R} 39=\mathrm{R} 41$, according to [6] the differential amplification factor of such amplifier can be expressed as follows:

$$
A_{\text {diff }}=\frac{R a}{R b}
$$

From the connection diagram in Figure 2 it is obvious that once the transistor T1 is fully closed, the gain of the amplifier should be as low as zero because both inputs of the differential amplifier are now fed with the same signal, provided the influence of the resistor R29 is negligible. Therefore the basic amplification factor is set by the resistor R35. The lower is the internal resistance of the transistor T1, the higher the amplification factor is because the attenuation of the signal at the negative input of the differential amplifier increases. The maximum gain of the amplifier is given by the value of the resistor R30. According to the parameters of the transistor T1, neglecting the output impedance of the previous stage and the resistivity of the resistor R25, the gain of this stage can be expressed as follows:

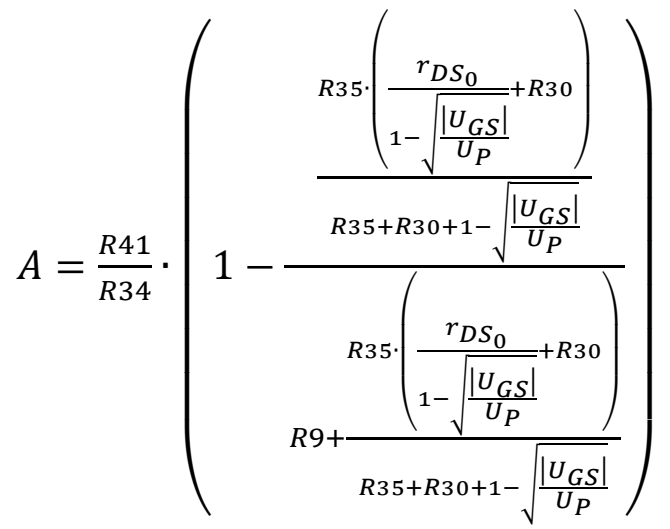

The meanings of the variables employed in (3) is obvious. R9, R30, R34, R35 and R41 refer to ohmic values of the relevant resistors in circuit diagram in the Figure 2. $\mathrm{U}_{\mathrm{GS}}$ refers to the gate-to-source voltage at the field effect transistor in Volts. For the proper function of the circuit it must be lower than $0 . U_{P}$ is the threshold voltage of the appropriate field effect transistor in Volts and $\mathrm{r}_{\mathrm{DS} 0}$ is the minimal internal resistance of the field effect transistor in Ohms. As can be seen, the amplification factor is dependent on the value of $U_{G S}$ and it is obvious that this dependency is not linear. With Maple, the function $\mathrm{A}\left(\mathrm{U}_{\mathrm{GS}}\right)$ for transistors with $\mathrm{U}_{\mathrm{P}}$ from -3 to $-2.5 \mathrm{~V}$ can be displayed according to Figure 4 . The meanings of the quantities displayed at the appropriate axes are described in the introduction to this paper. 
Because there exists an influence to the gate-to-source voltage $\mathrm{U}_{\mathrm{GS}}$ caused by processed signal waveforms, a perceptible distortion of the processed signal would be observed with no correction to cancel this phenomenon. According to [4], this distortion can be cancelled by establishing a signal feedback to the gate. In the circuit depicted in Figure 2 this is realized with the resistor R26.
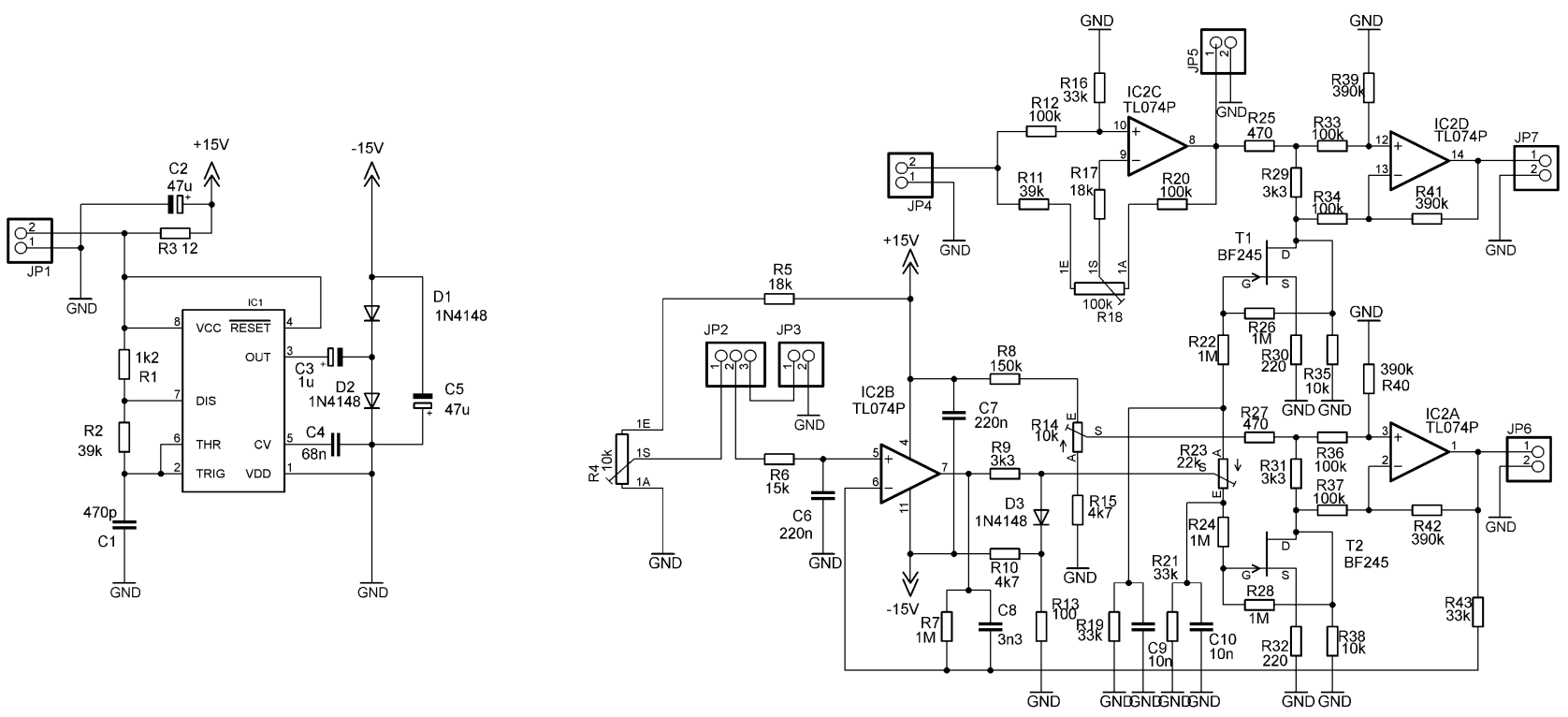

Fig. 2. Circuit diagram of the tested sample

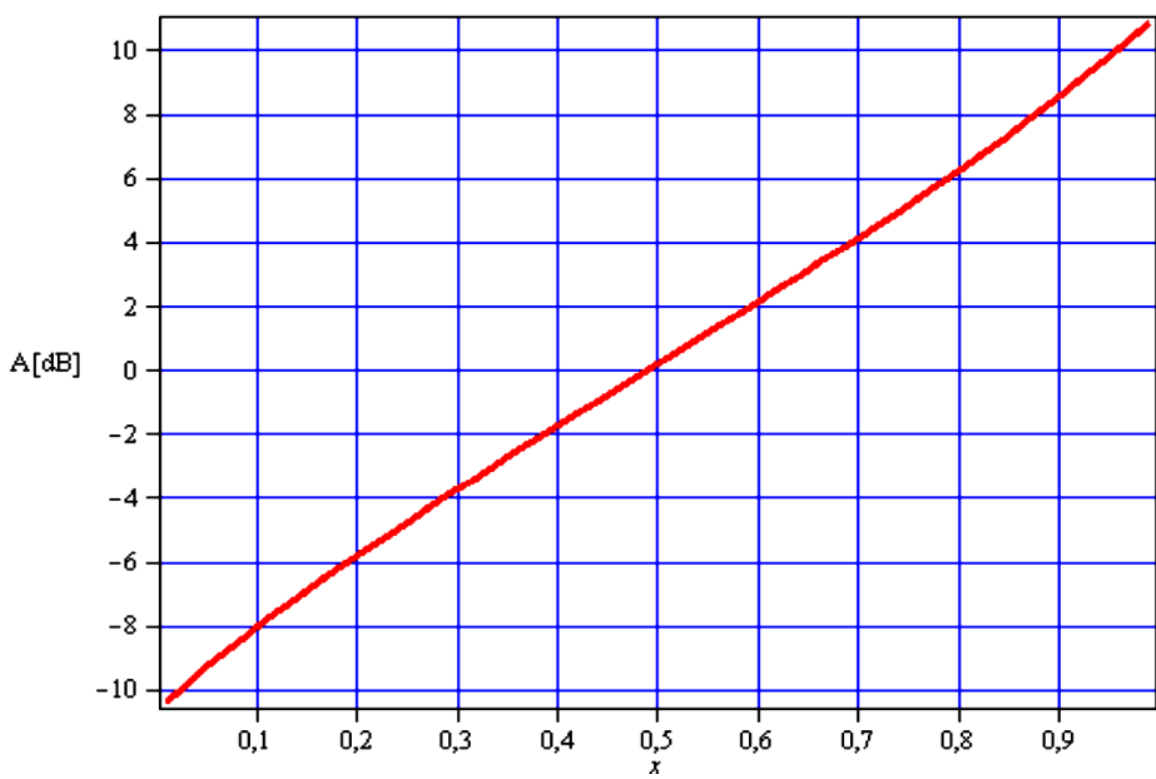

Fig. 3. Quasi-logarithmic dependence of the preamplifier's amplification factor on the linear potentiometer's setting (x)

\begin{tabular}{cl}
\hline Connector & Purpose \\
\hline JP1 & Power supply input (+ 15 V) \\
JP2 & Control voltage switch (Internal / External) \\
JP3 & External control voltage input \\
JP4 & Signal input \\
JP5 & Preamplifier's back-end (for service / measurement purposes) \\
JP6 & DC output of VCA II (for service / measurement purposes) \\
JP7 & Signal output \\
\hline
\end{tabular}

Table 1. Description of the connectors of the circuit depicted in Fig. 2 


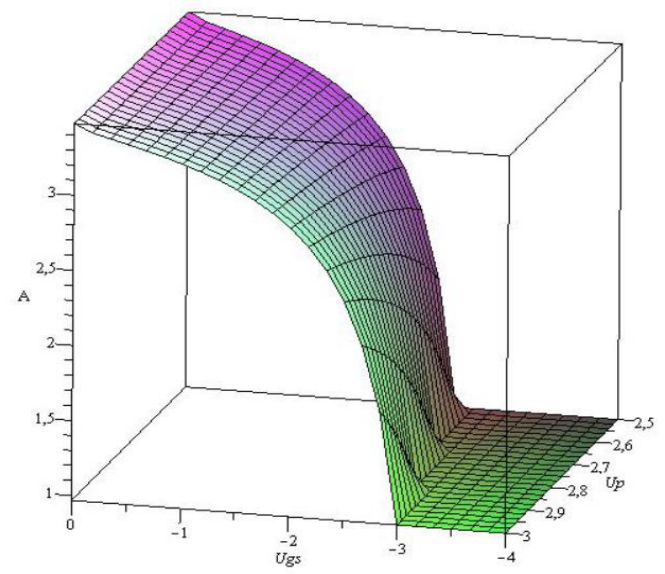

Fig. 4. Dependence of the VCA's amplification factor on UGS and Up (Maple model)

The VCA II amplifier is fed by a DC voltage of $1 \mathrm{~V}$, instead of any signal. This voltage is obtained from the lower supply voltage by a voltage divider composed of the resistors R8, R14 and R15. As R14 is a trimming resistor, the value of the reference voltage can be adjusted to the desired level. Its non-linear response to the controlling voltage is inverted to compensate the nonlinearity of the amplifier VCA I. The mechanism operates as follows: Let us assume that the amplifiers made with the operational amplifiers IC2D and IC2A are identical, which means that all used components are of zero value tolerances. Then the output voltage of the VCAII amplifier (IC2A) refers to the amplification factor set by the $\mathrm{U}_{\mathrm{GS}}$ voltage of the $\mathrm{T} 2$ transistor and this amplification factor indirectly corresponds to the amplification factor of the signal VCAI. The output of the auxiliary amplifier serves as a feedback for the DC input amplifier made with the operational amplifier IC2B. This DC amplifier sets the control voltage $\mathrm{U}_{\mathrm{GS}}$ of the transistors T1 and $\mathrm{T} 2$ to such level in which the output voltage of the auxiliary amplifier is equal to the input control voltage. In other words, if both, voltage controlled amplifiers are of equal characteristics, their amplification factor is equal to the control voltage connected to the PIN1 of JP3 connector or to the position of the slider of the potentiometer R4, depending on the setting of jumper JP2. Obviously, this dependence is only valid for the amplification factors achievable with the appropriate circuit construction. In this case $1 \mathrm{~V}$ control voltage refers to the amplification factor of $1(0 \mathrm{~dB})$ and $3.25 \mathrm{~V}$ refers to the amplification factor of $3.25(+10 \mathrm{~dB})$. To prevent driving the PN junctions of T1 and T2 transistor to their on-state in case the control voltage exceeded the upper boundary there is the diode D3 connected at the output of the IC2B operational amplifier. Its cathode is connected to a potential roughly equal to its junction potential so the transistors cannot be driven by voltages higher than zero. The rotary trimming resistor R23 serves to balance the small differences of the threshold voltages of the T1 and T2 transistors. However, for the proper function of the circuit these transistors should be paired and mounted close each to other in order their temperature was equal. The capacitors C9 and $\mathrm{C} 10$ are to cancel the influence of not-symmetrically-set rotary trimming resistor R14 to the feedbacks made with resistors R22, R26 and R24, R28. The capacitor C1 was added after the simulation experiences that proved that the feedback loop tends to oscillate.

\section{Achieved results}

The hereby described circuit has been constructed on a standalone printed circuit board in the form of a functional sample and its behavior was tested. The measured results were compared with the results of the simulation program Multisim. Due to the limited space of this paper, only the most interesting findings are described within this chapter.

\subsection{Preamplifier's frequency response at different gain settings}

The frequency response of the preamplifier based on the operating amplifier IC2C has been both simulated and measured. The results obtained by both methods are enlisted in the Table 2 . Together with the frequency response the course of the gain control of the preamplifier was checked, which is also reflected in the table.

\begin{tabular}{|c|c|c|c|c|c|c|}
\hline \multirow{3}{*}{ Frequency [Hz] } & \multicolumn{6}{|c|}{ Total Gain } \\
\hline & \multicolumn{2}{|c|}{ R18 set to MIN } & \multicolumn{2}{|c|}{ R18 set to MID } & \multicolumn{2}{|c|}{ R18 set to MAX } \\
\hline & Simulated & Measured & Simulated & Measured & Simulated & Measured \\
\hline 20 & -11.5 & -11.7 & 0 & 0 & +11.5 & +12.0 \\
\hline 50 & -11.5 & -11.7 & 0 & 0 & +11.5 & +12.0 \\
\hline 100 & -11.5 & -11.7 & 0 & 0 & +11.5 & +12.0 \\
\hline
\end{tabular}




\begin{tabular}{ccccccc}
\hline \hline 26TH DAAAM INTERNATIONAL S YMPOSIUM ON INTELLIGENT MANUFACTURING AND AUTOMATION \\
\hline \hline 200 & -11.5 & -11.7 & 0 & 0 & +11.5 & +12.0 \\
500 & -11.5 & -11.7 & 0 & 0 & +11.5 & +12.0 \\
1,000 & -11.5 & -11.7 & 0 & 0 & +11.5 & +12.0 \\
2,000 & -11.5 & -11.7 & 0 & 0 & +11.5 & +12.0 \\
5,000 & -11.5 & -11.7 & 0 & 0 & +11.5 & +12.0 \\
10,000 & -11.5 & -11.7 & 0 & 0 & +11.5 & +12.0 \\
20,000 & -11.5 & -11.53 & 0 & 0 & +11.5 & +12.0 \\
50,000 & -11.5 & -11.03 & 0 & +0.42 & +11.3 & +12.0 \\
100,000 & -11.5 & -9.77 & 0 & +0.98 & +10.9 & +12.34 \\
\hline
\end{tabular}

Table 2. Preamplifier's gain dependence on the control setting and frequency

\subsection{Response of the VCA to the controlling voltage}

The response of the VCA to the controlling voltage was simulated and measured within the control voltage range from 0 to $3.5 \mathrm{~V}$. While the simulation provided results close to the ideal response as depicted in Fig. 5, the measurements have shown negligible deviations.

The response of the voltage controlled amplifier to the control voltage was measured under the following circumstances:

- the adjustment of the trimming resistors was done as described in the text above,

- the input of the circuit was fed with a sinusoidal signal the frequency of which was $1 \mathrm{kHz}$ and the voltage of which was 1VRMS,

- the driving signal was connected to the connector JP4 and the output signal was measured at the connector JP7,

- no load was connected to the connector JP5,

- the control voltage was adjusted internally by the potentiometer R4,

- the gain of the preamplifier was set to $0 \mathrm{~dB}$.

Both, the measured and the ideal (simulated) responses of the VCA to the controlling voltage are depicted in the figure below.

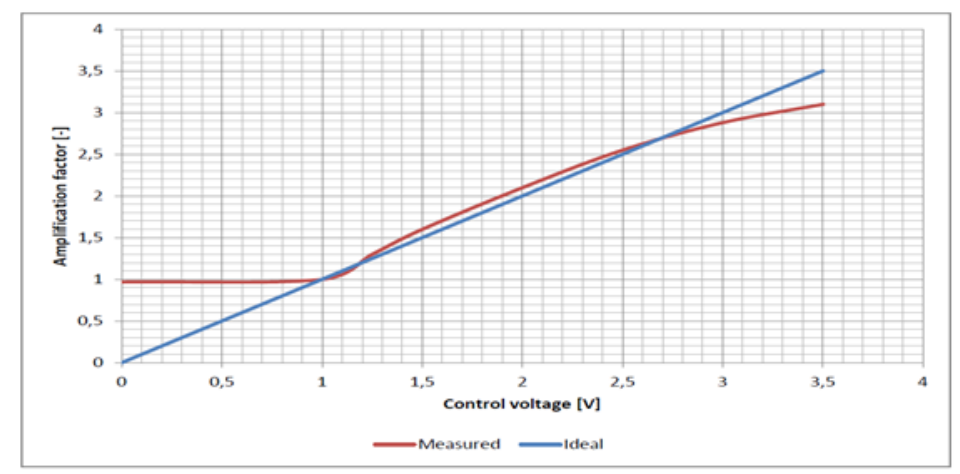

Fig. 5. Response of the VCA to the controlling voltage (measured and ideal/simulated course)

\subsection{Total harmonic distortion}

Whereas in the case of the above mentioned parameters the really measured values matched the simulated values with negligible deviations, in the case of the harmonic distortion the differences between the simulations and the really observed results were quite large.

The simulation of the total harmonic distortion of the VCA was run for the gain of $0,+3,+6$ and $+9 \mathrm{~V}$. The frequency of the input sinusoidal signal was set to $1 \mathrm{kHz}$ and its amplitude was set to $0 \mathrm{dBu}$. The total harmonic distortion was computed by means of Fourier transformation applied to the waveform at the output of the VCA. According to the simulation, the total harmonic distortion was below $0.01 \%$ in all cases.

The measurement procedure was the same as in case of the VCA's response to the control voltage measurement, but instead of voltmeter, an oscilloscope with Fourier analysis option was connected to the output connector JP7. The circuit was driven with a sinusoidal signal the frequency of which was $1 \mathrm{kHz}$ and the voltage of which was $1 \mathrm{~V}_{\mathrm{RMS}}$, which means that it was slightly overexcitated to the level of $+3 \mathrm{dBu}$. The second and the third harmonics at its output were observed, while the amplification factor was gradually increased from 1 to the maximum value. 
The measured results are enlisted in Table 3. The Table 3 includes information on the amplification factor of the VCA, the second and the third harmonics level in percents and the total overexcitation of the output of the circuit provided the nominal level is $0 \mathrm{dBu}$. The gain of the preamplifier was set to $0 \mathrm{~dB}$.

The third harmonic distortion is unacceptable, as the value of $8.2 \%$ at the gain of 1.5 is considerably high. This indicates that the linearization of the field effect transistor proposed in [4] does not work properly. Both harmonics disappear when the level of the driving signal is decreased to the maximum of 0.3VRMS. This indicates, that the VCA can be used well for the nominal level of input signal around - $10 \mathrm{dBu}$ in order to maintain a reasonable degree of ovrexcitation. However, the signal to noise ratio would be reduced accordingly. Another approach to cancelling the distortion is to use field effect transistors of a different type or to adjust the linearizing feedback properly.

\begin{tabular}{|c|c|c|c|}
\hline \multirow{2}{*}{$\begin{array}{c}\text { Control voltage } \\
\text { [V] }\end{array}$} & \multicolumn{2}{|c|}{ Harmonics } & \multirow{2}{*}{$\begin{array}{c}\text { Overexcitation } \\
{[\mathrm{dB}]}\end{array}$} \\
\hline & $2^{\text {nd }}[\%]$ & $3^{\text {rd }}[\%]$ & \\
\hline 1.0 & 1.0 & 3.1 & +3.0 \\
\hline 1.5 & 0.9 & 8.2 & +6.5 \\
\hline 2.0 & 0.7 & 3.1 & +9.0 \\
\hline 2.5 & 0.4 & 1.0 & +11.0 \\
\hline 3.0 & 0.1 & 0.4 & +12.5 \\
\hline
\end{tabular}

Table 3. Harmonic Distortion of the whole circuit according to the gain of the VCA.

\section{Conclusions}

Within this paper the description of the design of the voltage controlled amplifier for dynamics expanders is described. Its design was processed with the aid of software for mathematical computations and software for simulation of electrical circuits. Realistic models of components based on SPICE libraries were applied.

However, once the circuit was constructed in a form of a functional sample, using the same devices as applied in the simulations, several deviations from the expected behaviour were observed. Most of them were considered as negligible, but the amount of distortion produced at the VCA's gain setting between 1.0 and 2.0 is unacceptable.

As stated in the text of the paper, several attempts to eliminate the distortion are possible. The most feasible solution is to decrease the nominal level of the processed signal. However, this would lead to a commensurate increase of the noise of the circuit. Other possibilities consist in trying to find the field effect transistors that are more suitable for this purpose or in adjusting their linearizing feedback. These steps are planned to be realized on the new design version of the circuit. A solution based on switched capacitors [9] can be also taken in account as well as employing of transresistance amplifiers [10], [11].

\section{Acknowledgements}

This work was supported by the Ministry of Education, Youth and Sports of the Czech Republic within the National Sustainability Programme project No. LO1303 (MSMT-7778/2014).

\section{References}

[1] R. Dennis. Advance recording primer - dynamics signal processing, 2001. http://www.recordinginstitute. com/da154/ARP/chap3Sig/asp2.html.

[2] Dynamic signal processors. http://cfa.arizona.edu/studio/DynamicsProcessorsF08.pdf.

[3] M. Pospisilik, M. Adamek. Software-based audio signal dynamics expander. In Recent Researches in Circuits and Systems. 2012. ISBN: 978-1-61804-108-1.

[4] Vishay-Siliconix. Fets as voltage-controlled resistors. Tech. rep., Vishay - Siliconix, 1997.

[5] T1074 datasheet. http: //www.ece.usu.edu/ece_store/spec/TL074CN.pdf.

[6] J. Puncuchar. Operating amplifiers in electronics [Operacni zesilovace v elektronice]. BEN Technicka literatura, 5th edn., 2002. ISBN: 80-7300-059-8.

[7] P. Horowitz, W. Hill, The art of electronics, Second edition, Cambridge University Press.

[8] A. Motamed, H. Changku, M. Ismail, A low-voltage low-power wide-range CMOS variable gain amplifier, In IEEE Transactions on Circuits and Systems II: Analog and Digital Signal Processing, Vol. 45, Issue 7, ISSN: 1057-7130.

[9] P. E. Allen, E. Sanchez-Silencio, Switched Capacitor Circuits, Van Nostrand, New York, USA, 1984, ISBN: 97894-011-6991-2.

[10] A. Srinivasulu, P. S. Chandra. Grounded resistance/capacitance-controlled sinusoidal oscillators using operational transresistance amplifier. In WSEAS Transactions on Circuits and Systems, Vol. 13, pp. 145-152, 2014. E-ISSN: 2224-266X

[11] A. R. Pawade, R. D. Ghongade. Operational Transresistance Amplifier Using Submicron Technology. In International Journal of Advanced Research in Computer Science and Software Engineering. Volume 4, Issue 2, February 2014. ISSN: 2277 128X 